\title{
ON THE MECHANISM OF SEPARATION OF ETHANOL/WATER MIXTURES BY PERVAPORATION \\ II. EXPERIMENTAL CONCENTRATION PROFILES
}

\author{
M.H.V. MULDER, A.C.M. FRANKEN and C.A. SMOLDERS
}

Department of Chemical Technology, Twente University of Technology, P.0. Box 217, 7500 AE Enschede (The Netherlands)

(Received May 7, 1984; accepted in revised form September 25, 1984)

\section{Summary}

Ethanol-water concentration profiles in cellulose acetate membranes were measured under steady-state pervaporation conditions. Knowledge of these profiles leads to a better understanding of the diffusion process during pervaporation. The concentration profiles were determined by a film-stack method, using three to six layers. It is shown that permeation of ethanol-water mixtures proceeds in a coupled way and that crossterm diffusion coefficients need to be considered. Furthermore, the occurrence of sorption resistances at the feed/membrane interface can be established from these experiments.

\section{Introduction}

Separation of mixtures by pervaporation takes place by a solution-diffusion mechanism. In most cases coupled transport occurs, which means that the flux of a component in a mixture may be changed not only by the presence of other components but also by its own movement $[1,2]$. Because of the occurrence of coupling, it is generally not possible to predict membrane selectivities from parameters obtained from single component permeation experiments. Tock [3] demonstrated this for the separation of water-dioxane mixtures through a Nylon- 6 membrane.

Many investigators have made use of concentration dependent diffusion coefficients, but generally coupling in the solubility part was not considered. In most cases, ideal sorption was assumed [ 5-7], which means that a linear relationship should exist between the concentration of a component of a binary mixture inside the membrane and the concentration of that component in the liquid feed mixture. Recently, we showed that the assumption of ideal sorption cannot be used for ethanol-water mixtures, or in general, for non-ideal mixtures [8]. Therefore, coupling in the solubility part should also be taken into account.

Recently we proposed a solution-diffusion model, which takes into account coupling both in the solubility part and in the kinetic part [4]. The 
coupling was expressed in the equations for the flux, $J_{i}$, of component $i$ through $J_{i}=f\left\{S_{i}\left(c_{i}, c_{j}\right), D_{i}\left(c_{i}, c_{j}\right)\right\}$ where $S_{i}$ is the solubility and $D_{i}$ the diffusivity of component $i$.

The object of this paper is to present experimental concentration profiles for the system water (I)-ethanol (2)-cellulose acetate (3). Knowledge of these profiles permits the investigator to extract more appropriate data about diffusion in the pervaporation process. The concentration profiles were determined by a so-called film-stack method, which has been used by a number of other investigators [3,9-11]. In the previously described model [4], secondorder coupling effects were considered, but cross-terms in the flux equations were neglected. The results presented here permit us to verify this assumption.

\section{Theory}

\section{Single-component permeation}

If we assume that linear relations exist between fluxes and forces, where forces are written in terms of chemical potential gradients, then we can write for a diffusing component, $i$, through a membrane

$-J_{i}=L_{i} \nabla \mu_{i}$

For single-component permeation the generalized Fick's law can be derived from eqn. (1). Writing activities instead of chemical potentials eqn. (1) be comes

$$
-J_{i}=L_{i} R T \frac{\partial \ln a_{i}}{\partial c_{i}} \nabla c_{i}
$$

The activity, $a_{i}$, of a component in a polymer (or polymeric membrane) can be described by Flory-H uggins thermodynamics [12]. For the binary case, the activity of a component (index $i$ ) in the polymer (index $j$ ) is given by

$\ln a_{i}=\ln v_{i}+\left(1-\frac{V_{i}}{V_{j}}\right) v_{j}+\chi_{i j} v_{j}^{2}$

where $v_{i}$ is the volume fraction of penetrant, $v_{j}$ the volume fraction of polymer and $\chi_{i j}$ the Flory-Huggins interaction parameter (for this case, $\chi_{i j}$ is assumed to be concentration independent). If we define a diffusion coefficient, $D_{i}$, as

$D_{i}\left(c_{i}\right)=L_{i} R T \frac{\mathrm{a} \ln a_{i}}{\partial c_{i}}$

which for an ideal system $\left(V i=V_{j}\right.$ and $\left.\chi_{i j}=0\right)$ reduces to

$L_{i}=\frac{D_{i}\left(c_{i}\right) c_{i}}{R T}$ 
Combination of eqns. (2) and (4) gives

$-J_{i}=D_{i}\left(c_{i}\right) \nabla c_{i}$

Here $D_{i}$ is the diffusion coefficient of component $i$ in the polymer-fixed frame of reference. Diffusion coefficients of permeating components in polymers in general depend strongly on the state of swelling of the polymer, because of the plasticizing action of the liquid on the segmental motions. In the case of pervaporation, anisotropic swelling occurs in the direction from the upstream to the downstream side. At the upstream side the concentration is maximal while it is almost zero at the downstream side. Therefore, diffusion coefficients will vary considerably across the membrane.

Several expressions can be used to quantify the relationship between diffusion coefficient and concentration. Most authors have suggested an exponential relationship $[3,9,10,13-16]$.

$\mathbf{D}=D_{0} \exp (\gamma c)$

where $D_{0}$ is the diffusion coefficient at zero concentration of penetrant and $\gamma$ is a constant showing the plasticizing effect of the penetrant on the mobility of the penetrant in the membrane.

When combining eqns. (6) and (7) and integrating across the membrane using the following boundary conditions: $c_{i}=c_{0 i}$ at $x=0$ and $c_{i}=0$ at $x=1$, eqn. (8) is obtained

$J_{i}=\frac{D_{0 i}}{\gamma_{i} l}\left[\exp \left(\gamma_{i} c_{0 i}\right)-1\right]$

Defining a relative distance in the membrane $\left(x_{\mathrm{R}}=x / l\right)$, a substitution of this quantity into eqns. (6) and (7) gives

$-J_{i}=\frac{D_{0 i}}{l} \exp \left(\gamma_{i} c_{i}\right) \frac{\partial c_{i}}{\partial x_{\mathrm{R}}}$

and integration of eqn. (9) gives

$c_{i}=\frac{1}{\gamma_{i}} \ln \left[x_{\mathbf{R}}\left(1-\exp \left(\gamma_{i} c_{0 i}\right)\right)+\exp \left(\gamma_{i} c_{0 i}\right)\right]$

Note that the concentration profile (eqn. 10) does no longer contain diffusivity terms. When experimental concentration profiles are determined, $\gamma_{i}$ and $c_{0 i}$ can then be obtained from eqn. (10) and $D_{0 i}$ from steady-state permeation experiments with the help of eqn. (8).

Permeation of liquid mixtures

In the case of liquid mixtures, coupling phenomena should be taken into account. Recently, the following equation was used to describe the transport of binary liquid mixtures through polymeric membranes [4]; it can be ob- 
tained by combining eqns. (1) and (5):

$-J_{i}=\frac{c_{i} D_{i}(c)}{R T} \nabla \mu_{i}$

In eqn. (11) second-order effects are taken into account since the chemical potential of component $\mathrm{i}, \mu_{i}$, depends on the concentration of component $j$ and on the different interaction parameters used in the Flory-Huggins expression for a ternary system. Furthermore, the diffusion coefficient, $D_{i}$, is assumed to be dependent on the concentration of both component $i$ andj. In this approach, cross-term diffusion coefficients are assumed to be zero (or $D_{i i} / D_{i j, i \neq j} \gg 1$ ) and in a following section we will discuss if one is allowed to make this assumption.

If the concentration is expressed as a volume fraction, $\phi$, and if activities are used instead of chemical potentials, eqn. (11) becomes

$$
\begin{aligned}
& -J_{1}=\phi_{1} D_{1}\left(\phi_{1}, \phi_{2}\right)\left[\left(\frac{\partial \ln a_{1}}{\partial \phi_{1}}\right)_{\phi_{2}} \nabla \phi_{1}+\left(\frac{\partial \ln a_{1}}{\partial \phi_{2}}\right)_{\phi_{1}} \nabla \phi_{2}\right] \\
& -J_{2}=\phi_{2} D_{2}\left(\phi_{1}, \phi_{2}\right)\left[\left(\frac{\partial \ln a_{2}}{\partial \phi_{1}}\right)_{\phi_{2}} \nabla \phi_{1}+\left(\frac{\partial \ln a_{2}}{\partial \phi_{2}}\right)_{\phi_{1}} \nabla \phi_{2}\right]
\end{aligned}
$$

Equations (12) and (13) are identical to eqns. (18) and (19) of Ref. [4].

The partial derivatives $\partial \ln a_{1} / \partial \phi_{1}, \partial \ln a_{1} / \partial \phi_{2}, \partial \ln a_{2} / \partial \phi_{1}$ and $\partial \ln a_{2} / \partial \phi_{2}$ can be obtained from the Flory-Huggins equations for ternary systems (see Appendix). When data on experimental concentration profiles are available, values for the diffusion coefficients $\left(D_{1}\left(\phi_{1}, \phi_{2}\right)\right.$ and $\left.D_{2}\left(\phi_{1}, \phi_{2}\right)\right)$ can be obtained from eqns. (12) and (13).

\section{Experimental}

\section{Materials}

Cellulose acetate (E 398-3) was obtained from Eastman Chemicals. The solvents used were of analytical grade.

\section{Membrane preparation}

Polymer solutions were prepared by dissolving the polymer in a suitable solvent (acetone or dioxane). The membranes were prepared by casting the polymer solution onto a glass plate and allowing the solvent to evaporate in a nitrogen atmosphere. The membranes obtained were completely transparant.

\section{Pervaporation}

The pervaporation experiments were carried out in the apparatus shown in Fig. 1. A cross-section of the permeation cell is given in Fig. 2. This cell, which is a modification of the one described previously [20], has been developed in order to remove the membranes as quickly as possible. 


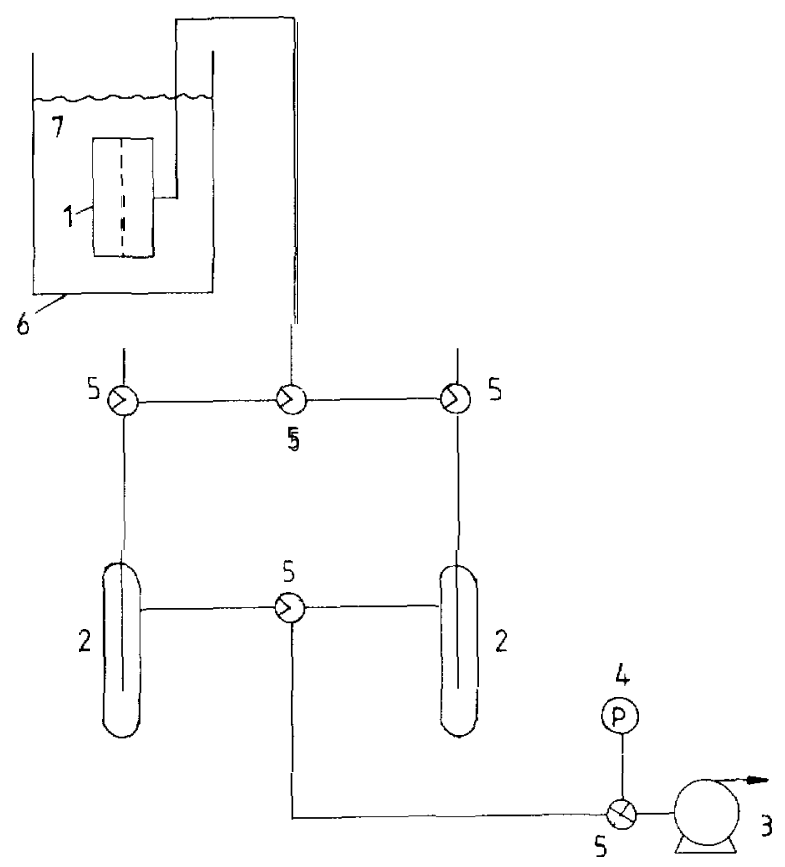

Fig. 1. Schematic presentation of the pervaporation apparatus: 1, permeation cell; 2, cold traps; 3, vacuum pump; 4, piranhi gauge; 5, two-way cocks; 6, jar; 7, liquid feed.

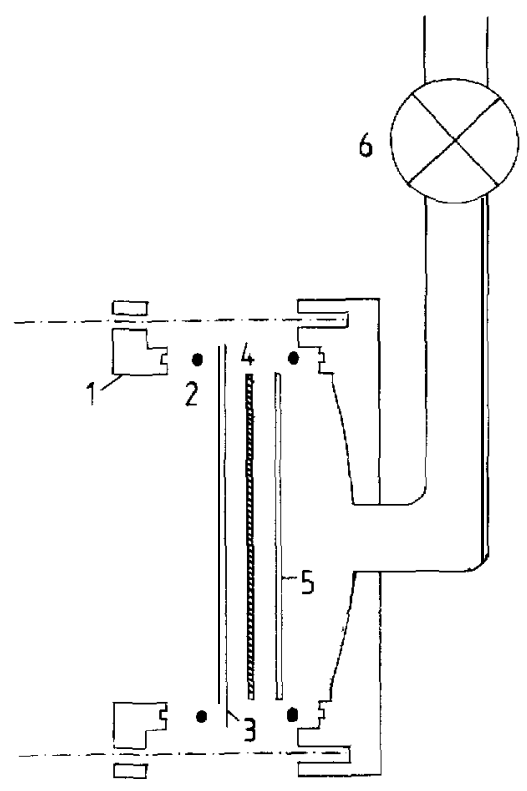

Fig. 2. Schematic presentation of the permeation cell: 1 , metal ring; 2 , gaskets; 3, membrane; 4 , porous metal filter; 5 , support disc; 6 , cock. 
Vacuum at the downstream side was maintained at a pressure of $13.3 \mathrm{~Pa}$ $(0.1 \mathrm{mmHg})$ by a Crompton Parkinson vacuum pump. The pressure was measured by an Edwards piranhi gauge. The concentration profiles were determined when steady-state conditions were reached. Product samples were taken at least every hour.

\section{Product analysis}

Analysis of binary ethanol-water mixtures was performed on a Varian model 3700 gas chromatograph fitted with a chromosorb 60/80 column and equipped with a thermal conductivity detector. For low ethanol concentrations (0-5\%), a flame ionization detector was used.

\section{Determination of the concentration profiles}

The multilayer membrane was prepared as follows: 3 to 6 individual layers, each of them with a thickness of at least $100 \mu \mathrm{m}$, were swollen in the liquid feed mixture. After equilibration, the layers were stacked one by one and the multilayer membrane was installed in the permeation cell. Preparing the multilayer membranes in this way resulted in negligible resistance at the interfaces of the different layers. This was checked by comparing permeabilities obtained from a multilayer membrane with those of a unilayer membrane of the same thickness. In both cases, the same permeation rates were obtained.

When steady-state conditions were reached, the permeation cell was removed from the jar, the surface was wiped quickly with tissue paper and the multilayer membrane was cut from the cell with a sharp knife. The individual layers were peeled off and immediately put in weighing tubes. The liquid present in each layer was removed from the membrane using a previously described distillation technique [8].

The amount of liquid was determined by weighing and the composition was determined by gas chromatography. The largest error was made in determining the concentration in the first layer because the time between removing the permeation cell from the liquid feed and putting the first layer in a weighing tube was about 1 minute. During this time desorption from the membrane occurs and the measured concentration is likely to differ from the actual concentration. In order to correct for this error, control experiments were carried out in which the weight decrease was measured as a function of time. The measured concentrations were corrected for the weight loss due to desorption. The second and subsequent layers were put in a weighing tube 5 to 10 seconds after each other and it was not necessary to correct the obtained results. The error in the mass balance was less than $5 \%$.

Results and discussion

Table 1 summarizes the results of the permeation experiments for the system water-cellulose acetate. The experimental concentration profile for 
TABLE 1

Permeation characteristics for the binary system water-cellulose acetate

\begin{tabular}{ll}
\hline Temperature & $17^{\circ} \mathrm{C}$ \\
$c_{\text {eq }}$, Equilibrium sorption & $0.125 \mathrm{~g} / \mathrm{g}$ \\
$c_{0}^{\mathrm{m}}$ & $0.125 \mathrm{~g} / \mathrm{g}$ \\
$J$, Permeation rate & $1.1 \times 10^{-3} \mathrm{~cm} / \mathrm{hr}$ \\
$l$, M embrane thickness & $500 \mu \mathrm{m}$ \\
Number of layers & $3-5$ \\
$D_{0}$ & $5.5 \times 10^{-9} \mathrm{~cm}^{2} / \mathrm{sec}$ \\
\hline
\end{tabular}

${ }^{a}$ From Ref. [8].

${ }^{b}$ Average of three experiments and then calculated for $500 \mu \mathrm{m}$ assuming inverse proportionality between permeation rate and membrane thickness.

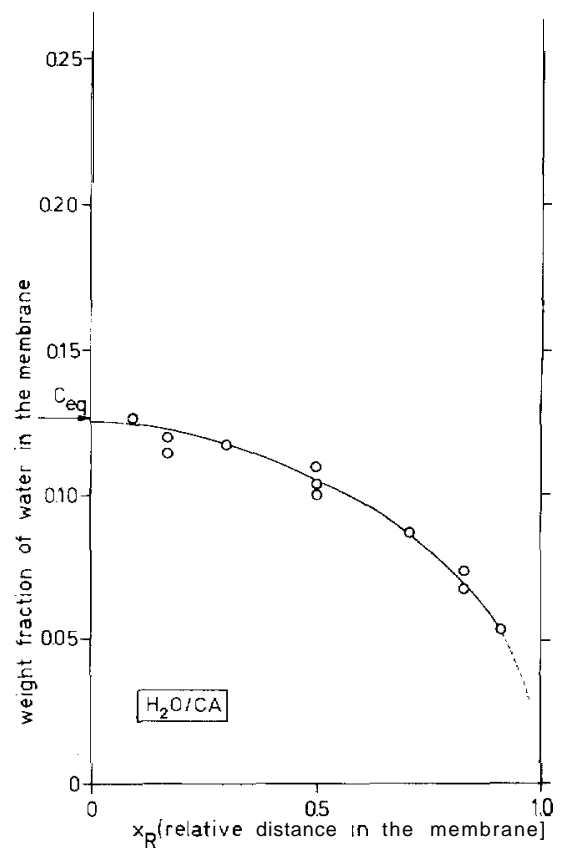

Fig. 3. Concentration profile of water in cellul ose acetate during steady-state pervaporation; $c_{\mathrm{eq}}$ (arrow) indicates the equilibrium sorption value.

this system is given in Fig. 3. The obtained profile is in agreement with the observations of Kim [10] on the same system. Another interesting feature from Fig. 3 is that the equilibrium sorption value (arrow in Fig. 3) and the concentration just inside the membrane during steady-state pervaporation are quite close. This indicates that the sorption resistance at the liquid/ polymer interface is negligible, and hence diffusion through the membrane is rate-determining.

Table 2 and Fig. 4 give the results obtained for the binary system ethanol- 
cellulose acetate. When comparing the results of the systems water-cellulose acetate and ethanol-cellulose acetate, one observes a number of differences:

(1) The permeation rate of water through cellulose acetate is much larger.

(2) The concentration profile of ethanol in cellulose acetate is much more concave, especially on the downstream half of the membrane.

(3) The difference between the equilibrium sorption value and the concentration just inside the membrane at the liquid/ polymer interface is considerable for the system ethanol-cellulose acetate.

(4) The $D_{0}$ value for ethanol is 50 times smaller than that for water.

TABLE 2

Permeation characteristics for the binary system ethanol-cellulose acetate

\begin{tabular}{ll}
\hline Temperature & $20^{\circ} \mathrm{C}$ \\
$c_{\text {eq }}$, Equilibrium sorption & $0.177 \mathrm{~g} / \mathrm{g}$ \\
$c_{0}^{\mathrm{m}}$ & $0.109 \mathrm{~g} / \mathrm{g}$ \\
$J$, Permeation rate & $7.8 \times 10^{-4} \mathrm{~cm} / \mathrm{hr}$ \\
$l$, Membrane thickness & $\mathrm{b}$ \\
Number of layers & $500 \mu \mathrm{m}$ \\
$D_{0}$ & $3.1 \times 10^{-10} \mathrm{~cm}^{2} / \mathrm{sec}$ \\
\hline
\end{tabular}

'From Ref. [8].

$\mathrm{b}$ Average of two experiments and then calculated for $500 \mu \mathrm{m}$ assuming inverse proportionality between permeation rate and membrane thickness.

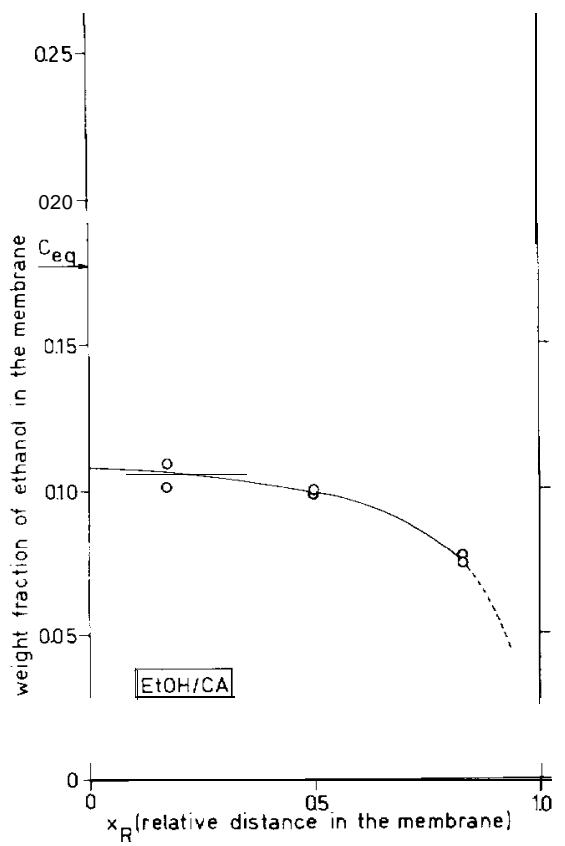

Fig. 4. Concentration profile of ethanol in cellulose acetate during steady-state pervaporation; $c_{\text {eq }}$ (arrow) indicates the equilibrium sorption value. 
For other systems also, (polyethylene-benzene and polyethylene-dioxane) considerable differences have been found between equilibrium sorption values and concentrations just inside the membrane [10].

The results for the ternary system water-ethanol-cellulose acetate are given in Table 3 and Figs. 5a-c. Again the difference between equilibrium sorption value and concentration just inside the membrane is considerable. When the individual profiles are considered, one can observe that both ethanol and water show a concentration drop at the liquid/membrane boundary. This observation is important because it clearly demonstrates the occurrence of a coupled sorption process. Furthermore, the ethanol and water profiles are rather similar with respect to the profiles of the pure components. Because the concentration profiles are known, the values for the diffusion coefficients of water and ethanol in cellulose acetate for the ternary system can be calculated from eqns. (12) and (13).

However, negative values are obtained for the diffusion coefficient of ethanol. This means that the simple approach of neglecting cross-term diffusion coefficients is not correct. The reason for these negative $D_{2}$ values can be explained by recalling eqns. (12) and (13). The term between the square brackets should have a negative value. Across the membrane from $\mathrm{x}=0$ to $\mathrm{x}=l$, the values of $\mathrm{d} \phi_{1} / \mathrm{d} x$ and $\mathrm{d} \phi_{2} / \mathrm{d} x$ are negative, as are also the partial derivatives $\partial \ln \alpha_{1} / \partial \phi_{2}$ and $\partial \ln a_{2} / \partial \phi_{1}$. The partial derivatives $\partial \ln a_{1} / \partial \phi_{1}$ and $\partial \ln a_{2} / \partial \phi_{2}$ are positive. Further, $\left|\partial \ln a_{i} / \partial \phi_{i}\right|>\left|\partial \ln a_{i} / \partial \phi_{j}\right|_{i \neq j}$. In the case of water (component 1$)$, there are no problems since $\left|\left(\partial \ln a_{1} / \partial \phi_{1}\right)\left(\mathrm{d} \phi_{1} / \mathrm{d} x\right)\right|>$ $\left|\left(\partial \operatorname{In} a_{1} / \partial \phi_{2}\right)\left(\mathrm{d} \phi_{2} / \mathrm{d} x\right)\right|$. Problems arise in the case of ethanol because, for low penetration distances, $\mathrm{d} \phi_{2} / \mathrm{d} x$ is very small (Fig. 5c) and since $\left|\mathrm{d} \phi_{1} / \mathrm{d} x\right|>$ $\left|\mathrm{d} \phi_{2} / \mathrm{d} x\right|$ while $\left|\partial \ln a_{2} / \partial \phi_{2}\right| \simeq\left|\partial \ln a_{2} / \partial \phi_{1}\right|$, the term between square brackets in eqn. (13) will have a positive value, resulting in a negative value for the

\section{TABLE 3}

Permeation characteristics for the ternary system water-ethanol-cellulose acetate

\begin{tabular}{|c|c|}
\hline $\begin{array}{l}\text { Temperature } \\
\text { Concentration of water in the feed } \\
c_{\text {eq }} \text {, Equilibrium sorption }{ }^{\mathrm{a}} \\
c_{0}^{\mathrm{m}} \text { (overall) } \\
J, \text { Permeation rate } \\
1 \text {, M embrane thickness } \\
\text { Number of layers } \\
c_{0}^{\mathrm{m}} \text { (water) }^{\mathrm{b}} \\
c_{\mathrm{eq}} \text { (water) }^{\mathrm{a}} \\
c_{0}^{\mathrm{m}} \text { (ethanol) }^{\mathrm{a}} \\
c_{\text {eq }} \text { (ethanol) } \\
\alpha \text {, Separation factor }\end{array}$ & $\begin{array}{l}17^{\circ} \mathrm{C} \\
35 \% \text { by weight } \\
0.253 \mathrm{~g} / \mathrm{g} \\
0.169 \mathrm{~g} / \mathrm{g} \\
1.6 \times 10^{-3} \mathrm{~cm} / \mathrm{h} \\
500 \mu \mathrm{m} \\
4-6 \\
0.100 \mathrm{~g} / \mathrm{g} \\
0.147 \mathrm{~g} / \mathrm{g} \\
0.069 \mathrm{~g} / \mathrm{g} \\
0.106 \mathrm{~g} / \mathrm{g} \\
9.8\end{array}$ \\
\hline
\end{tabular}

\footnotetext{
${ }^{\text {a }}$ From Ref. [8].

${ }^{b}$ Average of three experiments and then calculated for $500 \mu \mathrm{m}$ assuming inverse proportionality between permeation rate and membrane thickness.
} 

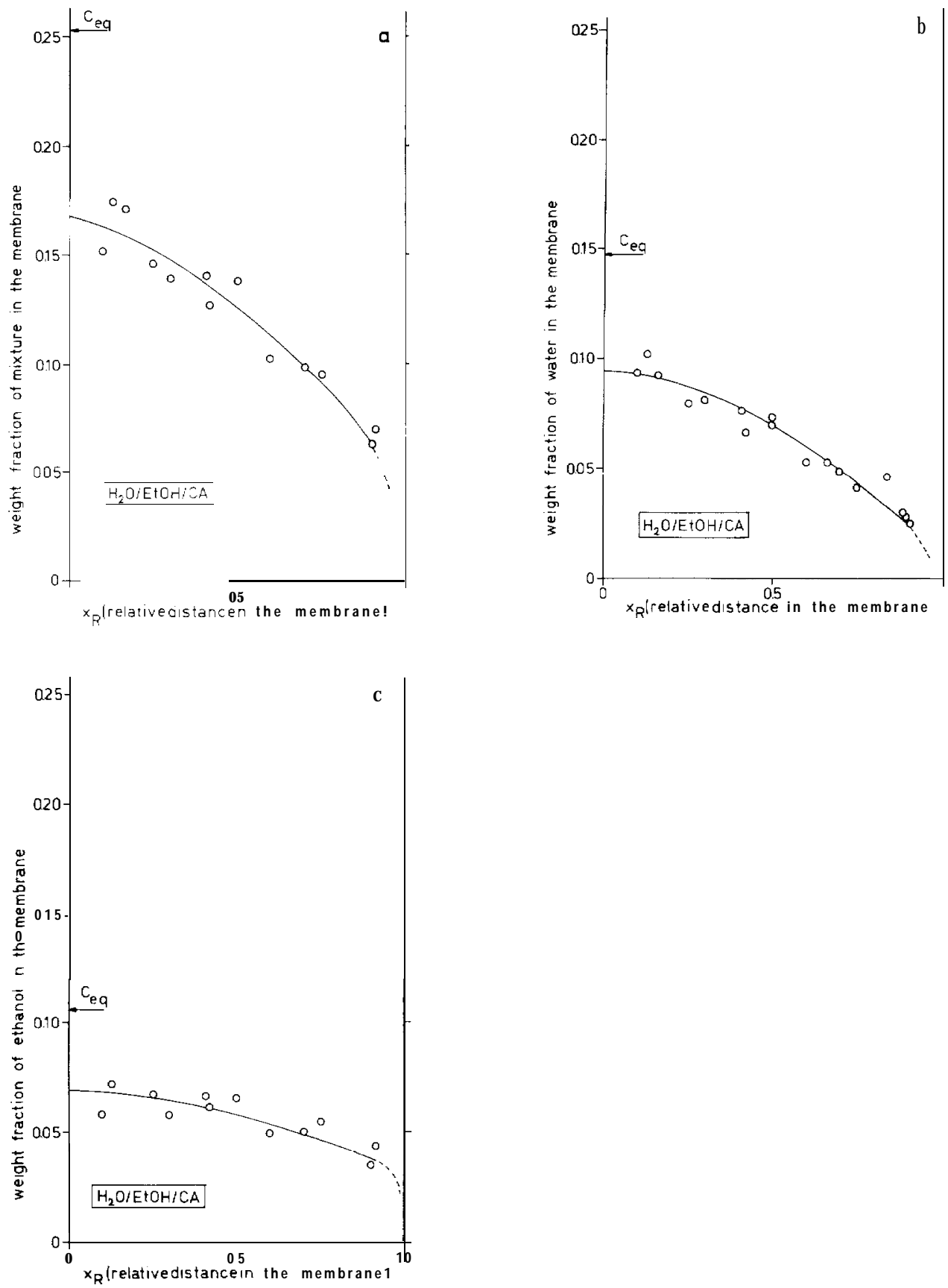

Fig. 5. Concentration profile of an ethanol-water mixture in cellulose acetate during steady-state pervaporation. Concentration in the feed: 35 wt.\% water; a, overall profile; $\mathrm{b}$, water profile; $\mathrm{c}$, ethanol profile. 
diffusion coefficient, $D_{2}$. Therefore, the simple phenomenological equation, eqn. (1), should be replaced by an equation where cross-term diffusion coefficients are taken into account.

Cussler [17] gave some empirical rules for deciding when multicomponent diffusion effects will be large. Two of these rules apply to the system water ethanol-cellulose acetate: (i) the components show strong thermodynamic interactions, and (ii) the concentration gradient of water in cellulose acetate is much different from that of ethanol in cellulose acetate. The equations used so far (eqns. 12 and 13) hold for the case where main-term diffusion coefficients are much larger than the cross-term diffusion coefficients $\left(D_{i i} \gg\right.$ $D_{i j, i \neq j}$ ). In a forthcoming article [18] we will report on experimental concentration profiles of o-xylene and p-xylene in cellulose propionate and discuss the results of this weakly interacting system using main-term diffusion coefficients only.

Taking cross-terms into account, eqn. (1) becomes

$-J_{j}=\sum_{j=1}^{n} \frac{D_{i j} c_{j}}{R T} \nabla \mu_{j}$

For steady-state transport of water and ethanol through a cellulose acetate membrane we now have

$-J_{1}=\frac{D_{11} c_{1}}{R T} \nabla \mu_{1}+\frac{D_{12} c_{2}}{R T} \nabla \mu_{2}$

$-J_{2}=\frac{D_{21} c_{1}}{R T} \nabla \mu_{1}+\frac{D_{22} c_{2}}{R T} \nabla \mu_{2}$

The first term on the right-hand side of eqn. (15) describes the flux of component 1 due to its own gradient and the second term of this equation describes the flux of component 1 due to the gradient of component 2 . This second term represents the coupling effect. In the system water-ethanolcellulose acetate, the cross-term diffusion coefficient $\left(D_{i j, i \neq j}\right)$ is likely to be a significant fraction of the main-term diffusion coefficient, especially in the case of component 2 (ethanol) where it dominates the effect of the main coefficient. This implies that the flux of ethanol due to the gradient of water should have a larger value than the flux of ethanol caused by its own gradient.

In general, the cross-term diffusion coefficients are not symmetric, i.e., $D_{12} \neq D_{21}$.

By expressing the concentration as a volume fraction and using activities instead of chemical potentials, eqns. (15) and (16) become

$$
\begin{aligned}
-J_{1}= & \frac{D_{11} \phi_{1}}{\Delta x}\left[\left(\frac{\partial \ln a_{1}}{\partial \phi_{1}}\right)_{\phi_{2}} \Delta \phi_{1}+\left(\frac{\partial \ln a_{1}}{\partial \phi_{2}}\right)_{\phi_{1}} \Delta \phi_{2}\right] \\
+ & \frac{D_{12} \phi_{2}}{\Delta x}\left[\left(\frac{\partial \ln a_{2}}{\partial \phi_{1}}\right)_{\phi_{2}} \Delta \phi_{1}+\left(\frac{\partial \ln a_{2}}{\partial \phi_{2}}\right)_{\phi} \Delta \phi_{2}\right]
\end{aligned}
$$




$$
\begin{aligned}
-J_{2} & =\frac{D_{21} \phi_{1}}{\Delta x}\left[\left(\frac{\partial \ln a_{1}}{\partial \phi_{1}}\right)_{\phi_{2}} \Delta \phi_{1}+\left(\frac{\partial \ln a_{1}}{\partial \phi_{2}}\right)_{\phi_{1}} \Delta \phi_{2}\right] \\
& +\frac{D_{22} \phi_{2}}{\Delta x}\left[\left(\frac{\partial \ln a_{2}}{\partial \phi_{1}}\right)_{\phi_{2}} \Delta \phi_{1}+\left(\frac{\partial \ln a_{2}}{\partial \phi_{2}}\right)_{\phi_{1}} \Delta \phi_{2}\right]
\end{aligned}
$$

Cussler [17] summarized different theories to quantify the cross-term diffusion coefficients. A general approach is to assume that the main-term diffusion coefficients $\left(D_{i i, i=1,2}\right)$ are closely related to the binary diffusion coefficients. These binary diffusion coefficients can be obtained from single-component permeation experiments, as given in Tables 1 and 2. The cross-diffusion coefficient can now be calculated from eqns. (17) and (18).

The ratios $\left|D_{12} / D_{11}\right|$ and $\left|D_{21} / D_{22}\right|$, or better $\left|\left(D_{12} / D_{11}\right)\left(\nabla \mu_{2} / \nabla \mu_{1}\right)\right|$ and $\left\{\left(D_{21} / D_{22}\right)\left(\nabla \mu_{1} / \nabla \mu_{2}\right) \mid\right.$ respectively, are a measure of the ternary effects and each of them is given in Figs. 6 and 7 as a function of the relative distance through the membrane, $x_{\mathrm{R}}$. Comparing Figs. 6 and 7 , one can see that the value of $\left|D_{21} / D_{22}\right|$ decreases when $x_{\mathrm{R}}$ increases, while the term $\mid\left(D_{21} / D_{22}\right)$ $\left(\nabla \mu_{1} / \nabla \mu_{2}\right) \mid$ increases in value. In fact, $\left|\left(D_{21} / D_{22}\right)\left(\nabla \mu_{1} / \nabla \mu_{2}\right)\right|$ is a better measure for multi-component diffusion effects than $\left|D_{21} / D_{22}\right|$ since the effects of the gradients are also involved. From Fig. 7 one can see that $\mid\left(D_{21} / D_{22}\right)\left(\nabla \mu_{1} \mid\right.$ $\left.\nabla \mu_{2}\right) \mid>1$, implying that the flux of component 2 (ethanol) due to the gradient of component 1 (water) has a larger value than the flux of component 2 caused by its own gradient. This is in agreement with the negative values for the diffusion coefficient, $D_{2}$, obtained from eqn. (13), implying that the assumption of using binary diffusion coefficients is not so bad after all. The flux of water is hardly affected by the gradient of ethanol, at least at

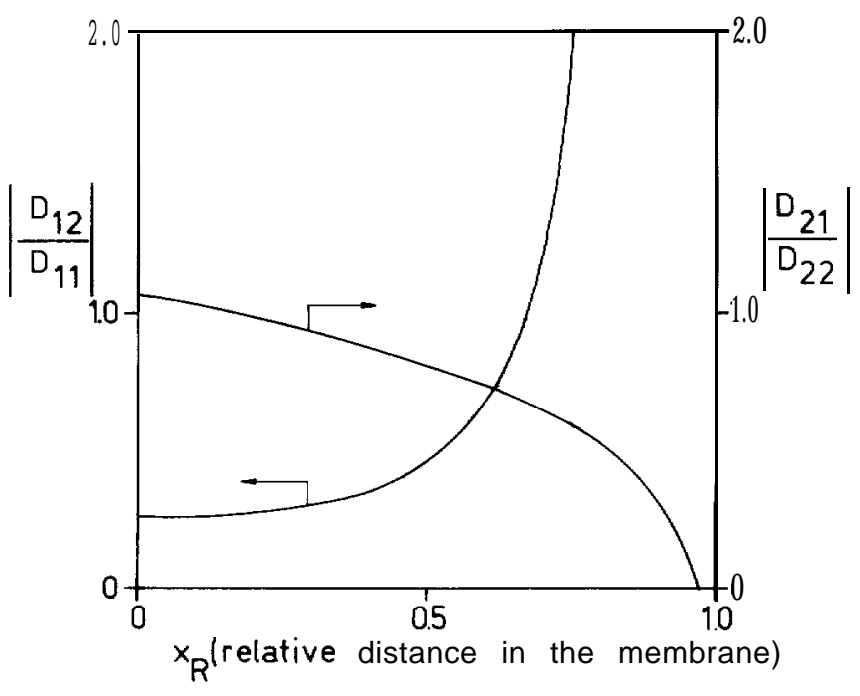

Fig. 6. Ratio of cross-term diffusion coefficients and main-term diffusion coefficients as a function of the relative distance in the membrane. 


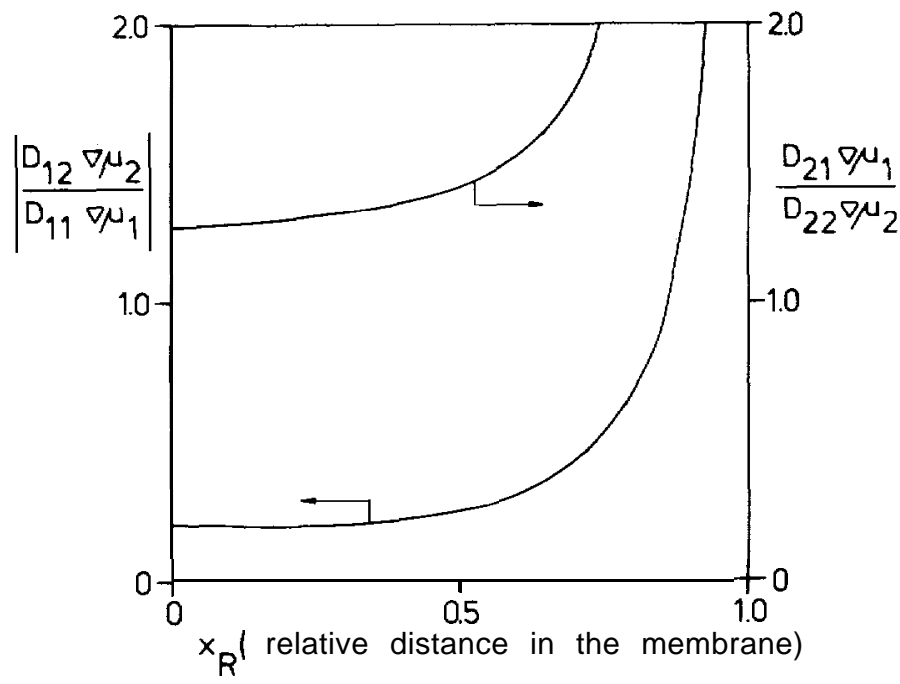

Fig. 7. Ratio of cross-term diffusion coefficient times gradient and main-term diffusion coefficient times gradient as a function of the relative distance in the membrane.

small penetration distances $\left(x_{R}<0.5\right)$. At high penetration distance, coupling effects become more and more important.

The results presented here demonstrate beyond any doubt the occurrence of coupling phenomena. Because it has been assumed that the main-term diffusion coefficient is simply equal to the binary diffusion coefficient, it is hardly possible to draw quantitative conclusions about the magnitude of the coupling effect.

\section{In terfacial resistance}

Interfacial resistances exist when there is no thermodynamic equilibrium at the feed/membrane and membrane/permeate interfaces. This means that the chemical potential of a component $i$ in the feed is not equal to the chemical potential of component $i$ just inside the membrane.

Hwang [19] showed that the interfacial resistance contributes to a large extent to the total resistance during the permeation of dissolved oxygen in water through a silicone rubber membrane.

By investigating the concentration profiles of the system water-ethanolcellulose acetate (pure components and mixture) the occurrence of boundary resistances could be demonstrated (see Figs. 4 and 5). Because of the nonideality of the various systems, activity profiles are preferred over concentration profiles. In Figs. 8 and 9, the activity profiles for the systems water cellulose acetate and ethanol-cellulose acetate are given. These activity profiles have been calculated from the experimental concentration profiles using Flory-Huggins thermodynamics. The $\chi_{i j}$ parameters (eqn. 3), which for these systems are assumed to be constant, were taken from Ref. [4] (see Table 4). In the case of water, no resistance at the feed/membrane interface 


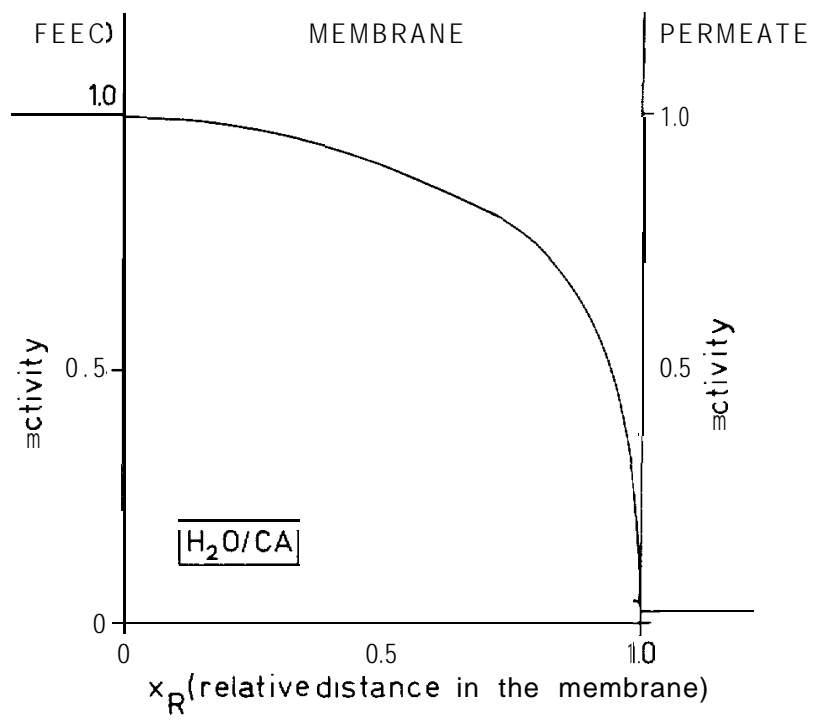

Fig. 8. Activity profile for a binary system water-cellulose acetate.

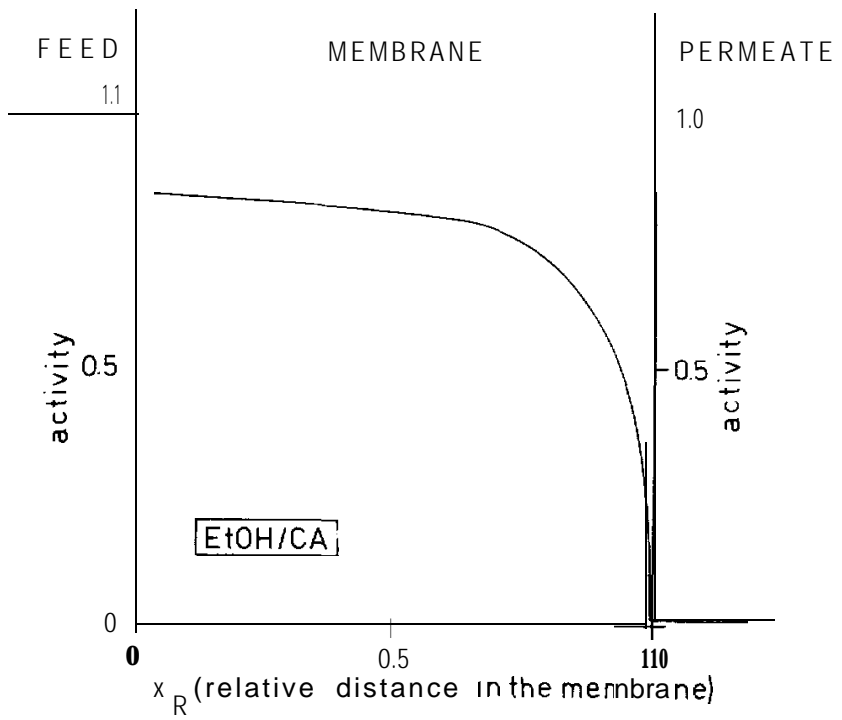

Fig. 9. Activity profile for the binary system ethanol-cellulose acetate.

\section{TABLE 4}

Interaction parameters for the system water (1)-ethanol (2)-cellulose acetate (3) (taken from Ref. [4])

\begin{tabular}{ll}
\hline$\chi_{12}$ & $0.98-1.35 v_{2}+4.15 v_{2}^{2}-3.31 v_{2}^{3}+0.89 v_{2}^{4}$ \\
$\chi_{13}$ & 1.4 \\
$\chi_{23}$ & 1.1 \\
\hline
\end{tabular}


can be observed (Fig. 8) while in the case of ethanol there is an appreciable resistance. Comparing Fig. 4 with Fig. 9, one can see that the activity drop is much smaller than the concentration drop.

Recently [4] it was assumed that equality of chemical potential existed at the feed/membrane interface (and at the membrane/permeate interface). The results presented here clearly demonstrate that this assumption does not necessarily hold.

\section{Conclusions}

The transport of ethanol-water mixtures through cellulose acetate membranes cannot be described with a simple phenomenological model where cross-term diffusion coefficients are neglected. Therefore, multicomponent effects should be considered. Cross-term diffusion coefficients appear to be a significant fraction of the main-term diffusion coefficients and, in the case of ethanol diffusing through cellulose acetate in the presence of water, the cross-term effect even dominates the main-term effect.

Boundary resistances found in pervaporation are caused by sorption phenomena and, in the case of permeation of liquid mixtures, these sorption resistances are coupled too.

\section{Acknowledgement}

This paper is based upon work financially supported by the Ministeries van Economische Zaken, en Onderwijs en Wetenschappen in The Netherlands.

\section{List of symbols}

$\begin{array}{ll}a & \text { Activity } \\ D & \text { Diffusion coefficient }\left(\mathrm{cm}^{2}-\mathrm{sec}^{-1}\right) \\ D_{0} & \text { Diffusion coefficient at zero concentration }\left(\mathrm{cm}^{2}-\mathrm{sec}^{-1}\right) \\ D_{i i} & \text { Main-term diffusion coefficient }\left(\mathrm{cm}^{2}-\mathrm{sec}^{-1}\right) \\ D_{i j, i \neq j} & \text { Cross-term diffusion coefficient }\left(\mathrm{cm}^{2}-\mathrm{sec}^{-1}\right) \\ J & \text { Permeation rate }\left(\mathrm{cm}-\mathrm{hr} r^{-1}\right) \\ c_{\mathrm{eq}} & \text { Equilibrium sorption value (weight fraction) } \\ c_{0}^{\mathrm{m}} & \text { Concentration inside the membrane at } \mathrm{x}=0 \text { (weight fraction) } \\ l & \text { Membrane thickness }(\mathrm{cm}) \\ L & \left.\text { Phenomenological coefficient (cm-mol- } \mathrm{sec}^{-1}-N^{-1}\right) \\ R & \text { Gas constant }\left(J-m o l^{-1}-\mathrm{K}^{-1}\right) \\ T & \text { Temperature }(\mathrm{K}) \\ v & \text { Volume fraction in the binary mixture } \\ V & \text { Molar volume }\left(\mathrm{cm}^{3}-\mathrm{mol}^{-1}\right) \\ x_{\mathrm{R}} & \text { Relative thickness in the membrane } \\ \mathrm{Ax} & \text { Thickness of an infinitesimal thin layer }(\mathrm{cm})\end{array}$




$\begin{array}{ll}\alpha & \text { Separation factor } \\ \gamma & \text { Exponential factor } \\ \chi & \text { Flory-Huggins interaction parameter } \\ \phi & \text { Volume fraction in the ternary system } \\ \mu & \text { Chemical potential }\left(\mathrm{J}-\mathrm{mol}^{-1}\right)\end{array}$

$\begin{array}{ll}\text { indices } & \\ 1 & \text { Water } \\ 2 & \text { Ethanol } \\ 3 & \text { Cellulose acetate } \\ i & \text { Component } i \\ j & \text { Component } \mathrm{j}\end{array}$

\section{References}

1 P. Meares, The physical chemistry of transport and separation by membranes, in: P. Meares (Ed.), Membrane Separation Processes, Elsevier, Amsterdam, 1976, Chap. 1.

2 P. Meares, Transport through polymer membranes from the liquid phase, Ber. Bunsenges. Phys. Chem., 83 (1979) 342.

3 R.W.M. Tock, J.Yu. Cheung and R.L. Cook, Dioxane-water transport through Nylon6 membranes, Sep. Sci., 9 (1974) 361.

4 M.H.V. Mulder and C.A. Smolders, On the mechanism of separation of ethanol/ water mixtures by pervaporation. I. Calculation of concentration profiles, J. Membrane Sci., 17 (1984) 289.

5 A.B. Krewinghaus, Solution and transport of organic liquids and vapors in structurally modified polyethylene, Ph.D. Thesis, Massachusetts Institute of Technology, A pril 1966.

6 F.W. Greenlaw, R.A. Shelden and E.V. Thompson, Dependence of diffusive permeation rates on upstream and downstream pressures. II. Two component permeant, J. Membrane Sci., 2 (1977) 333.

7 R. Rautenbach and R. Albrecht, Separation of organic binary mixtures by pervaporation, J. Membrane Sci., 7 (1980) 203.

8 M.H.V. Mulder, A.C.M. Franken and CA. Smolders, Preferential sorption versus preferential permeability in pervaporation, J. Membrane Sci., 22 (1985) 155.

9 P. Aptel, J. Cuny, J. Jozefowicz, G. Morel and J. Neel, Liquid transport through membranes prepared by grafting of polar monomers onto poly( tetrafluoroethylene) films. II. Some factors determining pervaporation rate and selectivity, J. Appl. Polym. Sci., 18 (1974) 351.

10 S.N. Kim and K. Kammermeyer, Actual concentration profiles in membrane separation, Sep. Sci., 5 (1970) 679.

11 S. Rosenbaum and 0. Cotton, Steady-state distribution of water in cellulose acetate membrane, J. Polym. Sci., A-I, 7 (1969) 101.

12 P.J. Flory, Principles of Polymer Chemistry, Cornell University Press, Ithaca, N ew York, 1953.

13 R.Y.M. Huang and V.J.C. Lin, Separation of liquid mixtures by using polymer membranes. I. Permeation of binary organic liquid mixtures through polyethylene, J. Appl. Polym. Sci., 12 (1968) 2615.

14 R.B. Long, Liquid permeation through plastic films, Ind. Eng. Chem., Fundam., 4 (1965) 445. 
15 H.W. Chandler and E.J. Henley, Diffusion and equilibrium studies for the system acrylonitrile-styrene-polyethylene, AIChE J., 7 (196 1) 295.

16 D.W. McCall, Diffusion in ethylene polymers, I. Desorption kinetics for a thin slab, J. Polym. Sci., 26 (1957) 151.

17 E.L. Cussler, M ulticomponent D iffusion, Elsevier, A msterdam, 1976.

18 M.H.V. Mulder, A.C.M. Franken and C.A. Smolders, Separation of isomeric xylenes. A fundamental approach, to be published in J. Membrane Sci.

19 S.T. H wang, T.E.S. T ang and K. K ammermeyer, Transport of dissolved oxygen through silicone rubber membrane, A mer. Chem. Soc., Polym. Prepr., 10 (1969) 978.

20 M.H.V. Mulder, F. Kruitz and C.A. Smolders, Separation of isomeric xylenes by pervaporation through cellulose ester membranes, J. M embrane Sci., 11 (1982) 349.

\section{Appendix}

The Gibbs free energy of mixing for a ternary system is given by the following relationship :

$\frac{\Delta G_{\mathrm{m}}}{R T}=n_{1} \ln \phi_{1}+n_{2} \ln \phi_{2}+n_{3} \ln \phi_{3}+\chi_{12}\left(u_{2}\right) n_{1} \phi_{2}+\chi_{13} n_{1} \phi_{3}+\chi_{23} n_{2} \phi_{3}$

The subscripts refer to water (1), ethanol (2) and cellulose acetate (3); $n_{i}$ and $\phi_{i}$ are the number of moles and the volume fraction of component $i$, respectively, $\chi_{12}$ is the water-ethanol interaction parameter and it is assumed to be a function of $u_{2}$ with $u_{2}=\phi_{2} /\left(\phi_{1}+\phi_{2}\right) ; \chi_{13}$ and $\chi_{23}$ are the water-cellulose acetate and ethanol-cellulose acetate parameters, respectively, and these are assumed to be concentration independent. The values of the different interaction parameters, which have been taken from Ref. [4], are given in Table 4.

From eqn. $(\mathrm{Al})$, the following relationships for the chemical potentials of the components in the polymer can be derived:

$$
\begin{aligned}
\ln a_{1}= & \ln \phi_{1}+\left(1-\phi_{1}\right)-\phi_{2}\left(\frac{V_{1}}{V_{2}}\right)-\phi_{3}\left(\frac{V_{1}}{V_{3}}\right)+\left(\chi_{12}\left(u_{2}\right) \phi_{2}+\chi_{13} \phi_{3}\right)\left(\phi_{2}+\phi_{3}\right) \\
& -\chi_{23} \frac{V_{1}}{V_{2}} \phi_{2} \phi_{3}-u_{1} u_{2} \phi_{2} \frac{\partial \chi_{12}}{\partial u_{2}}
\end{aligned}
$$

$$
\ln a_{2}=\ln \phi_{2}+\left(1-\phi_{2}\right)-\phi_{1}\left(\frac{V_{2}}{V_{1}}\right)-\phi_{3}\left(\frac{V_{2}}{V_{3}}\right)
$$

$$
+\left(\chi_{12}\left(u_{2}\right) \phi_{1} \frac{V_{2}}{V_{1}}+\chi_{23} \phi_{3}\right)\left(\phi_{1}+\phi_{3}\right)-\chi_{13}\left(\frac{V_{2}}{V_{1}}\right) \phi_{1} \phi_{3}+\left(\frac{V_{2}}{V_{1}}\right) u_{1}^{2} \phi_{2} \frac{\partial \chi_{12}}{\partial u_{2}}
$$

$$
\begin{aligned}
\frac{\mathrm{a} \ln a_{1}}{\partial \phi_{1}}= & \frac{1}{\phi_{1}}-1+\frac{V_{1}}{V_{2}}-\chi_{12} \phi_{2}+\chi_{23} \phi_{2} \frac{V_{1}}{V_{2}}-\chi_{13}\left(2-2 \phi_{1}-\phi_{2}\right) \\
& +\phi_{2}\left(1-\phi_{1}\right) \frac{\partial \chi_{12}}{\partial \phi_{1}}+u_{2}^{2}\left(1-2 u_{2}\right) \frac{\partial \chi_{12}}{\partial u_{2}}+u_{1} u_{2}^{3} \frac{\partial^{2} \chi_{12}}{\partial u_{2}^{2}}
\end{aligned}
$$




$$
\begin{aligned}
\frac{\partial \ln a_{1}}{\partial \phi_{2}}= & -\frac{V_{1}}{V_{2}}+\frac{V_{1}}{V_{3}}+\chi_{12}\left(1-\phi_{1}\right)+\chi_{13}\left(\phi_{1}-1\right)+\chi_{23} \frac{V_{1}}{\bar{V}_{2}}\left(2 \phi_{2}+\phi_{1}-1\right) \\
& +\phi_{2}\left(1-\phi_{1}\right) \frac{\partial \chi_{12}}{\partial \phi_{2}}-2 u_{1}^{2} u_{2} \frac{\partial \chi_{12}}{\partial u_{2}}-u_{1}^{2} u_{2}^{2} \partial^{2} \chi_{12} \\
\frac{\partial \ln a_{2}}{\partial \phi_{1}}= & -\frac{V_{2}}{V_{1}}+\frac{V_{2}}{V_{3}}+\chi_{12} \frac{V_{2}}{V_{1}}\left(1-\phi_{2}\right)+\chi_{13} \frac{V_{2}}{V_{1}}\left(2 \phi_{1}+\phi_{2}-1\right) \\
& -\chi_{23}\left(1-\phi_{2}\right)+\frac{V_{2}}{V_{1}} \phi_{1}\left(1-\phi_{2}\right) \frac{\partial \chi_{12}}{\partial \phi_{1}}+2 \frac{V_{2}}{V_{1}} u_{1} u_{2}^{2} \frac{\partial \chi_{12}}{\partial u_{2}} \\
& -\frac{V_{2}}{V_{1}} u_{1}^{2} u_{2}^{2} \frac{\partial^{2} \chi_{12}}{\partial u_{2}^{2}} \\
& \frac{1}{\phi_{2}}-1+\frac{V_{2}}{V_{3}}-\chi_{12} \frac{V_{2}}{V_{1}} \phi_{1}+\chi_{13} \frac{V_{2}}{V_{1}} \phi_{1}+\chi_{23}\left(2 \phi_{2}+\phi_{1}-2\right) \\
& +\frac{V_{2}}{V_{1}} \phi_{1}\left(1-\phi_{2}\right) \frac{\partial \chi_{12}}{\partial \phi_{2}}+\frac{V_{2}}{V_{1}} u_{1}^{2}\left(1-2 u_{2}\right) \frac{\partial \chi_{12}}{\partial u_{2}} \\
& +\frac{V_{2}}{V_{1}} u_{1}^{3} u_{2} \frac{\partial^{2} \chi_{12}}{\partial u_{2}^{2}}
\end{aligned}
$$

